\title{
Momentum-Space Approach to Asymptotic Expansion for Stochastic Filtering *
}

\author{
Masaaki Fujii
}

1st version: September 10, 2012/ Current version: March 24, 2013

\begin{abstract}
This paper develops an asymptotic expansion technique in momentum space for stochastic filtering. It is shown that Fourier transformation combined with a polynomial-function approximation of the nonlinear terms gives a closed recursive system of ordinary differential equations (ODEs) for the relevant conditional distribution. Thanks to the simplicity of the ODE system, higher order calculation can be performed easily. Furthermore, solving ODEs sequentially with small sub-periods with updated initial conditions makes it possible to implement a substepping method for asymptotic expansion in a numerically efficient way. This is found to improve the performance significantly where otherwise the approximation fails badly. The method is expected to provide a useful tool for more realistic financial modeling with unobserved parameters, and also for problems involving nonlinear measure-valued processes.
\end{abstract}

Keywords Zakai equation · polynomial-function approximation · measure-valued process

\section{Introduction}

In many areas, researchers frequently encounter the situation where crucial parameters for their models are not directly observable in our mother nature or in experiments. This is particularly the case, for example, in engineering, applied physics, finance and economics. To get the best estimate of the unobservable from what we can directly observe is the goal of stochastic filtering. The most famous example with analytical solution is Kalman-Bucy filter [Bucy (1959), Kalman (1960), Kalman and Bucy (1961)], which assumes that both of the signal and observation processes are linear and hence is associated with Gaussian distribution.

However, there are many cases where interested variables follow nonlinear stochastic processes and their distributions are far from Gaussian. This is particularly the case for financial problems. In fact, many people were forced to realize the sheer impacts of non-Gaussianity in the last financial crisis followed by the collapse of Lehman Brothers. Researchers, practitioners as well as regulators now clearly recognize the importance of not only the first two moments but also every other detail of the relevant

* The manuscript is accepted for publication in the Annals of the Institute of Statistical Mathematics. The official manuscript will be available in http://www.ism.ac.jp/editsec/aism-e.html

Masaaki Fujii

Faculty of Economics, The University of Tokyo, 7-3-1, Hongo, Bunkyo-ku, Tokyo, 113-0033 Japan

E-mail: mfujii@e.u-tokyo.ac.jp 
distribution. Here, we need to deal with nonlinear filtering problems. Filtering theory has a long history and is still developing very rapidly, partly helped by the great increase of computational power. Recently, there appeared a thick volume edited by Crisan and Rozovskii (2011) from Oxford university press, which contains latest developments and reviews for theoretical as well as numerical techniques for nonlinear filtering problems. In particular, the article written by Frey and Runggaldier in the same volume gives a nice review of applications to various financial valuation problems under partial observation.

In this paper, we propose a simple approximation scheme based on an asymptotic expansion method in momentum space for nonlinear filtering problems. The method should be also useful for other financial problems that do not require filtering. Widely used "position-space" asymptotic expansion method (See, Takahashi (1999), and references therein.) is transformed into a simpler form in the momentum (or Fourier transformed) apace, and the resultant dynamics of the characteristic function is given by a closed recursive system of ordinary differential equations (ODEs). It is shown that the form of ODEs unchanged for any order of expansion, which allows straightforward numerical implementation for higher order approximations. Furthermore, dividing the original time horizon into a set of small sub-periods and solving the ODEs sequentially with updated initial conditions, which we call substepping method for asymptotic expansion, increase the parameter space where the approximation is effective. Two simple examples are discussed to demonstrate how the method works. We also make a brief comment on the possibility that the same method can be used to analyze other measure-valued nonlinear systems.

\section{Preliminaries for Nonlinear Filtering}

\subsection{Zakai equation}

Let $(\Omega, \mathcal{F}, \mathbb{P})$ be a probability space with a filtration $\left(\mathcal{F}_{t}\right)_{t \geq 0}$ satisfying the usual conditions. We consider $n$-dimensional signal process $X=\left\{X_{t}, t \geq 0\right\}$ and $m$-dimensional observation process $Y=\left\{Y_{t}, t \geq 0\right\}$ following the dynamics of

$$
\begin{aligned}
& d X_{t}=\mu_{t}\left(X_{t}\right) d t+\eta_{t}\left(X_{t}\right) d V_{t}+\bar{\eta}_{t}\left(X_{t}\right) d W_{t} \\
& d Y_{t}=h_{t}\left(X_{t}\right) d t+d W_{t}
\end{aligned}
$$

with $Y_{0}=0$ and an independent initial distribution for $X_{0}$. Here $V$ and $W$ are independent $(\mathbb{P}, \mathcal{F})$-Brownian motions with dimensionality $d$ and $m$, respectively. $\mu$, $h, \eta$ and $\bar{\eta}$ are deterministic functions of $(t, x)$ and take values in $\mathbb{R}^{n}, \mathbb{R}^{m}, \mathbb{R}^{n \times d}$ and $\mathbb{R}^{n \times m}$, respectively. The functions $\mu, \eta$ and $\bar{\eta}$ are assumed to satisfy appropriate conditions so that (1) has a unique solution. The measurable function $h$ is assumed to satisfy the conditions that make the following process $Z=\left\{Z_{t}, t \geq 0\right\}$ be a martingale:

$$
Z_{t}=\exp \left(-\int_{0}^{t} h_{s}\left(X_{s}\right)^{\top} d W_{s}-\frac{1}{2} \int_{0}^{t}\left\|h_{s}\left(X_{s}\right)\right\|^{2} d s\right)
$$

where $T$ denotes the transposition. We denote $\left\{\mathcal{Y}_{t}, t \geq 0\right\}$ be the usual augmented filtration generated by the process $Y$. Our goal of the filtering problem is to obtain the conditional distribution $\pi_{t}$ of the signal $X$ at time $t$ given the information available from observing the process $Y$ in the interval of $[0, t]$. In other words, for a given arbitrary bounded function $\varphi$, compute

$$
\pi_{t}(\varphi)=\mathbb{E}\left[\varphi\left(X_{t}\right) \mid \mathcal{Y}_{t}\right]
$$


Let us define the measure $\widetilde{\mathbb{P}}$ by

$$
\left.\frac{d \widetilde{\mathbb{P}}}{d \mathbb{P}}\right|_{\mathcal{F}_{t}}=Z_{t}
$$

and the associated inverse relation

$$
\left.\frac{d \mathbb{P}}{d \widetilde{\mathbb{P}}}\right|_{\mathcal{F}_{t}}=\widetilde{Z}_{t}
$$

where $\widetilde{Z}_{t}=Z_{t}^{-1}$ can be written as

$$
\widetilde{Z}_{t}=\exp \left(\int_{0}^{t} h_{s}\left(X_{s}\right)^{\top} d Y_{s}-\frac{1}{2} \int_{0}^{t}\left\|h_{s}\left(X_{s}\right)\right\|^{2} d s\right)
$$

Note that the process $Y$ becomes a standard $(\widetilde{\mathbb{P}}, \mathcal{F})$-Brownian motion.

We define the unnormalized conditional distribution of $X$ to be the measure-valued process $\rho=\left\{\rho_{t}, t \geq 0\right\}$

$$
\rho_{t}(\varphi)=\widetilde{\mathbb{E}}\left[\widetilde{Z}_{t} \varphi\left(X_{t}\right) \mid \mathcal{Y}_{t}\right] \quad \widetilde{\mathbb{P}}-\text { a.s. }
$$

which is $\mathcal{Y}_{t}$-adapted and càdlàg. Here, $\widetilde{\mathbb{E}}[\cdot]$ denotes the expectation in the measure $\widetilde{\mathbb{P}}$. The desired filtered density function can then be obtained from the relation

$$
\pi_{t}(\varphi)=\frac{\rho_{t}(\varphi)}{\rho_{t}(\mathbf{1})}
$$

It is known that the dynamics of $\rho$ satisfies the following Zakai equation for arbitrary smooth bounded function $\varphi$ :

$$
\rho_{t}(\varphi)=\rho_{0}(\varphi)+\int_{0}^{t} \rho_{s}\left(A_{s} \varphi\right) d s+\int_{0}^{t} \rho_{s}\left(\left(h_{s}+B_{s}\right)^{\top} \varphi\right) d Y_{s}
$$

with initial value $\rho_{0}(\varphi)=\mathbb{E}\left[\varphi\left(X_{0}\right)\right]$ associated with a given distribution of $X_{0}$. Here, $A_{s}$ is the infinitesimal generator of $X$ at time $s$

$$
A_{s}=\sum_{i=1}^{n} \mu_{s}^{i}(x) \frac{\partial}{\partial x_{i}}+\frac{1}{2} \sum_{i, j=1}^{n}\left(\eta_{s} \eta_{s}^{\top}(x)+\bar{\eta}_{s} \bar{\eta}_{s}^{\top}(x)\right)_{i, j} \frac{\partial^{2}}{\partial x_{i} \partial x_{j}}
$$

and

$$
B_{s}^{k}=\sum_{i=1}^{n}\left(\bar{\eta}_{s}^{\top}(x)\right)_{k, i} \frac{\partial}{\partial x_{i}}, \quad k=1, \cdots, m
$$

For the derivation of the Zakai equation and the other technical details, see Bain and Crisan (2008), for example. The goal of this paper is to develop a simple scheme to solve the Zakai equation (3). 


\subsection{Filtered Characteristic Function}

Let us consider a function

$$
\psi(\xi, x)=\exp \left(i \xi^{\top} x\right)
$$

with $\xi, x \in \mathbb{R}^{n}$, where $i=\sqrt{-1}$. If one obtains the conditional expectation of this function, i.e.,

$$
\pi_{t}(\psi(\xi, \cdot))=\frac{\widetilde{\mathbb{E}}\left[\exp \left(i \xi^{\top} X_{t}\right) \widetilde{Z}_{t} \mid \mathcal{Y}_{t}\right]}{\widetilde{\mathbb{E}}\left[\widetilde{Z}_{t} \mid \mathcal{Y}_{t}\right]}
$$

for each $\xi$, one can derive the conditional expectation for an arbitrary choice of $\varphi$. This fact can be seen as follows: Let us consider the inverse Fourier transformation $\phi_{t}(\cdot)$

$$
\phi_{t}(z)=\frac{1}{(2 \pi)^{n}} \int_{\mathbb{R}^{n}} e^{-i \xi^{\top} z} \pi_{t}(\psi(\xi, \cdot)) d^{n} \xi
$$

which can be evaluated as

$$
\begin{aligned}
\phi_{t}(z) & =\frac{1}{(2 \pi)^{n}} \int_{\mathbb{R}^{d}} \frac{1}{\widetilde{\mathbb{E}}\left[\widetilde{Z}_{t} \mid \mathcal{Y}_{t}\right]} \widetilde{\mathbb{E}}\left[\exp \left(i \xi^{\top}\left(X_{t}-z\right)\right) \widetilde{Z}_{t} \mid \mathcal{Y}_{t}\right] d^{n} \xi \\
& =\frac{\widetilde{\mathbb{E}}\left[\delta\left(X_{t}-z\right) \widetilde{Z}_{t} \mid \mathcal{Y}_{t}\right]}{\widetilde{\mathbb{E}}\left[\widetilde{Z}_{t} \mid \mathcal{Y}_{t}\right]},
\end{aligned}
$$

where $\delta(\cdot)$ denotes a $n$-dimensional Dirac delta function. The above function actually corresponds to the conditional density of the $X_{t}$ since

$$
\begin{aligned}
\int_{\mathbb{R}^{d}} \varphi(z) \phi_{t}(z) d^{n} z & =\frac{\widetilde{\mathbb{E}}\left[\varphi\left(X_{t}\right) \widetilde{Z}_{t} \mid \mathcal{Y}_{t}\right]}{\widetilde{\mathbb{E}}\left[\widetilde{Z}_{t} \mid \mathcal{Y}_{t}\right]} \\
& =\pi_{t}(\varphi) .
\end{aligned}
$$

Therefore, $\left\{\pi_{t}(\psi(\xi))\right\}$ and equivalently $\left\{\rho_{t}(\psi(\xi))\right\}$ contain all the important information one needs. In fact, many of the financial valuation problems with partial information end up with calculating an integration with the conditional density $\phi_{t}(d z)$ (See, Frey and Runggaldier (2011) for a review.).

\section{Asymptotic Expansion in Momentum Space}

From the discussion in the previous section, we want to solve the Zakai equation for the characteristic function $\rho_{t}(\psi(\xi))$. Although one can directly approximate the Zakai equation for a specific problem, it would be uneconomical. This is because the form of Zakai equation depends on the target function $\varphi$ for which one takes conditional expectation even in a common signal-observation system. 


\subsection{Perturbed System}

In order to make the system tractable, we replace the parameters in the original system in (1) and (2) by

$$
\begin{array}{ll}
\mu_{t}(x) \rightarrow f_{t}+\epsilon F_{t}(x) & \eta_{t}(x) \rightarrow \nu_{t}+\epsilon \sigma_{t}(x) \\
\bar{\eta}_{t}(x) \rightarrow \epsilon \gamma_{t}(x) & h_{t}(x) \rightarrow \epsilon H_{t}(x)
\end{array}
$$

and consider the $n$-dimensional signal and the $m$-dimensional observation processes as

$$
\begin{aligned}
& d X_{t}^{(\epsilon)}=\left(f_{t}+\epsilon F_{t}\left(X_{t}^{(\epsilon)}\right)\right) d t+\left(\nu_{t}+\epsilon \sigma_{t}\left(X_{t}^{(\epsilon)}\right)\right) d V_{t}+\epsilon \gamma_{t}\left(X_{t}^{(\epsilon)}\right) d W_{t} \\
& d Y_{t}=\epsilon H_{t}\left(X_{t}^{(\epsilon)}\right) d t+d W_{t}
\end{aligned}
$$

Here, $f_{t} \in \mathbb{R}^{n}$ and $\nu_{t} \in \mathbb{R}^{n \times d}$ are deterministic functions of time and $\nu_{t} \nu_{t}^{\top}$ is assumed to be positive definite. Note that the signal process is now a function of $\epsilon$, which is emphasized by a superscript " $(\epsilon)$ ". As one can see, in the limit of $\epsilon \downarrow 0$, the system yields a free decoupled Gaussian signal process for which the density function is exactly known. In the following, we try to expand the conditional density around it by taking into account state-dependent and observation effects perturbatively.

As explained in the previous section, we are interested in the unnormalized distribution

$$
\rho^{(\epsilon)}(\psi(\xi))=\widetilde{\mathbb{E}}\left[\widetilde{Z}_{t}^{(\epsilon)} \psi\left(\xi, X_{t}^{(\epsilon)}\right) \mid \mathcal{Y}_{t}\right]
$$

with

$$
\widetilde{Z}_{t}^{(\epsilon)}=\exp \left(\epsilon \int_{0}^{t} H_{s}\left(X_{s}^{(\epsilon)}\right)^{\top} d Y_{s}-\frac{\epsilon^{2}}{2} \int_{0}^{t}\left\|H_{s}\left(X_{s}^{(\epsilon)}\right)\right\|^{2} d s\right)
$$

and

$$
\left.\frac{d \mathbb{P}}{d \widetilde{\mathbb{P}}}\right|_{\mathcal{F}_{t}}=\widetilde{Z}_{t}^{(\epsilon)}
$$

The corresponding Zakai equation becomes

$$
\rho_{t}^{(\epsilon)}(\psi(\xi))=\rho_{0}(\psi(\xi))+\int_{0}^{t} \rho_{s}^{(\epsilon)}\left(A_{s}^{(\epsilon)} \psi(\xi)\right) d s+\epsilon \int_{0}^{t} \rho_{s}^{(\epsilon)}\left(\left(H_{s}+B_{s}\right)^{\top} \psi(\xi)\right) d Y_{s}
$$

where conditional distribution $\rho$ is now also a function of $\epsilon$. Here,

$$
B_{s}^{k}=\sum_{i=1}^{n}\left(\gamma_{s}^{\top}(x)\right)_{k, i} \frac{\partial}{\partial x_{i}}, \quad k=1, \cdots, m .
$$

and the infinitesimal generator is given by

$$
\begin{aligned}
A_{s}^{(\epsilon)}= & \sum_{i=1}^{n}\left(f_{s}^{i}+\epsilon F_{s}^{i}(x)\right) \frac{\partial}{\partial x_{i}}+\sum_{i, j=1}^{n} \frac{1}{2}\left(\nu_{s}+\epsilon \sigma_{s}(x)\right)\left(\nu_{s}+\epsilon \sigma_{s}(x)\right)_{i j}^{\top} \frac{\partial^{2}}{\partial x_{i} \partial x_{j}} \\
& +\sum_{i, j=1}^{n} \epsilon^{2} \frac{1}{2}\left(\gamma_{s}(x) \gamma_{s}(x)^{\top}\right)_{i j} \frac{\partial^{2}}{\partial x_{i} \partial x_{j}} .
\end{aligned}
$$

Our goal is to expand

$$
\rho_{t}^{(\epsilon)}(\psi(\xi))=\rho_{t}^{[0]}(\psi(\xi))+\epsilon \rho_{t}^{[1]}(\psi(\xi))+\epsilon^{2} \rho_{t}^{[2]}(\psi(\xi))+\cdots
$$

and obtain $\rho_{t}^{[j]}(\psi(\xi)), j=\{0,1,2, \cdots\}$ up to an arbitrary order. Here, we have defined

$$
\rho_{t}^{[j]}(\psi(\xi)):=\left.\frac{1}{j !} \frac{\partial^{j}}{\partial \epsilon^{j}} \rho_{t}^{(\epsilon)}(\psi(\xi))\right|_{\epsilon=0} .
$$


3.2 Recursive system for Asymptotic Expansion

We now expand the Zakai equation for each order of $\epsilon$. Note that, for any polynomial function $G$ of $x$, one can write

$$
G(x) e^{i \xi^{\top} x}=G\left(D_{\xi}\right) e^{i \xi^{\top} x}
$$

where, $G\left(D_{\xi}\right)$ denotes the differential operator obtained by replacing each $x_{j}$ in the function by $\left(D_{\xi}\right)_{j}$, which is a derivative operator defined as

$$
D_{\xi}=\frac{\partial}{i \partial \xi} \text {. }
$$

This fact allows one to write

$$
\rho_{t}^{(\epsilon)}(G \psi(\xi))=G\left(D_{\xi}\right) \rho_{t}^{(\epsilon)}(\psi(\xi))
$$

which is linear for $\rho_{t}^{(\epsilon)}(\psi(\xi))$.

In order to avoid nonlinearity, we make use of this property of polynomial functions. With slight abuse of notations, we treat $F_{t}(x), \sigma_{t}(x), \gamma_{t}(x)$ and $H_{t}(x)$ as arbitrary accurately approximated polynomial functions of $x$ (and time) for the corresponding original functions. By Weierstrass' polynomial approximation theorem, this is always possible for any continuous function within a closed interval. In practice, one can take wide enough interval within which the signal process resides with probability sufficiently close to one and an associated polynomial approximation accurate enough for that range.

Then, one can formally write

$$
A_{s}^{(\epsilon)} \psi(\xi, x)=\left(A_{s}^{[0]}(\xi)+\epsilon A_{s}^{[1]}\left(\xi, D_{\xi}\right)+\epsilon^{2} A_{t}^{[2]}\left(\xi, D_{\xi}\right)\right) \psi(\xi, x)
$$

where

$$
\begin{aligned}
& A_{s}^{[0]}(\xi)=i \xi^{\top} f_{s}-\frac{1}{2} \xi^{\top}\left(\nu_{s} \nu_{s}^{\top}\right) \xi \\
& A_{s}^{[1]}\left(\xi, D_{\xi}\right)=i \xi^{\top} F_{s}\left(D_{\xi}\right)-\frac{1}{2} \operatorname{tr}\left[\xi \xi^{\top}\left(\nu_{s} \sigma_{s}^{\top}\left(D_{\xi}\right)+\sigma_{s}\left(D_{\xi}\right) \nu_{s}^{\top}\right)\right] \\
& A_{s}^{[2]}\left(\xi, D_{\xi}\right)=-\frac{1}{2} \operatorname{tr}\left[\xi \xi^{\top}\left(\sigma_{s}\left(D_{\xi}\right) \sigma_{s}^{\top}\left(D_{\xi}\right)+\gamma_{s}\left(D_{\xi}\right) \gamma_{s}^{\top}\left(D_{\xi}\right)\right)\right]
\end{aligned}
$$

and similarly

$$
\left(H_{s}(x)+B_{s}(x)\right)^{\top} \psi(\xi, x)=\left(H_{s}^{\top}\left(D_{\xi}\right)+i \xi^{\top} \gamma_{s}\left(D_{\xi}\right)\right) \psi(\xi, x) .
$$

Substituting (5), (6) and (7) into the Zakai equation (4), one can easily confirm the following result.

Theorem 1 An arbitrary order of the asymptotic expansion $\rho_{t}^{[j]}(\psi(\xi)), j \in\{0,1,2, \cdots\}$

$$
\rho_{t}^{(\epsilon)}(\psi(\xi))=\rho_{t}^{[0]}(\psi(\xi))+\epsilon \rho_{t}^{[1]}(\psi(\xi))+\epsilon^{2} \rho_{t}^{[2]}(\psi(\xi))+\cdots
$$

of the unnormalized filtered characteristic function

$$
\rho_{t}^{(\epsilon)}(\psi(\xi))=\widetilde{\mathbb{E}}\left[\exp \left(i \xi^{\top} X_{t}^{(\epsilon)}\right) \widetilde{Z}_{t}^{(\epsilon)} \mid \mathcal{Y}_{t}\right]
$$


satisfies

$$
\begin{aligned}
d \rho_{t}^{[j]}(\psi(\xi))= & A_{t}^{[0]}(\xi) \rho_{t}^{[j]}(\psi(\xi)) d t \\
& +\left\{A_{t}^{[1]}\left(\xi, D_{\xi}\right) \rho_{t}^{[j-1]}(\psi(\xi))+A_{t}^{[2]}\left(\xi, D_{\xi}\right) \rho_{t}^{[j-2]}(\psi(\xi))\right\} d t \\
& +\left(H_{t}^{\top}\left(D_{\xi}\right)+i \xi^{\top} \gamma_{t}\left(D_{\xi}\right)\right) \rho_{t}^{[j-1]}(\psi(\xi)) d Y_{t}
\end{aligned}
$$

with initial conditions $\rho_{0}^{[0]}(\psi(\xi))=\rho_{0}(\psi(\xi)), \rho_{0}^{[j]}(\psi(\xi))=0(j \geq 1)$ and the convention that

$$
\rho^{[k]}(\psi(\xi)) \equiv 0
$$

for $k<0$.

Considering a special case where there is no observation, one obtains a simple corollary for a standard unconditional characteristic function.

Corollary 1 An arbitrary order of the asymptotic expansion $\rho_{t}^{[j]}(\psi(\xi)), j \in\{0,1,2, \cdots\}$

$$
\rho_{t}^{(\epsilon)}(\psi(\xi))=\rho_{t}^{[0]}(\psi(\xi))+\epsilon \rho_{t}^{[1]}(\psi(\xi))+\epsilon^{2} \rho_{t}^{[2]}(\psi(\xi))+\cdots
$$

of the characteristic function

$$
\rho_{t}^{(\epsilon)}(\psi(\xi))=\mathbb{E}\left[\exp \left(i \xi^{\top} X_{t}^{(\epsilon)}\right)\right]
$$

satisfies

$$
\begin{aligned}
d \rho_{t}^{[j]}(\psi(\xi))= & A_{t}^{[0]}(\xi) \rho_{t}^{[j]}(\psi(\xi)) d t \\
& +\left\{A_{t}^{[1]}\left(\xi, D_{\xi}\right) \rho_{t}^{[j-1]}(\psi(\xi))+A_{t}^{(2)}\left(\xi, D_{\xi}\right) \rho_{t}^{[j-2]}(\psi(\xi))\right\} d t
\end{aligned}
$$

with initial conditions $\rho_{0}^{[0]}(\psi(\xi))=\rho_{0}(\psi(\xi)), \rho_{0}^{[j]}(\psi(\xi))=0(j \geq 1)$ and the convention that

$$
\rho^{[k]}(\psi(\xi)) \equiv 0
$$

for $k<0$.

The above result shows that one only has to deal with a set of decoupled ODEs in terms of momentum $\{\xi\}$ with a given observation path of $Y$. It is straightforward to solve the above equation for each $\xi$ up to a certain $\epsilon$-order, and use discrete Fourier transformation technique to obtain the density function. In Fourier analysis of smooth functions, it is well-known that most of the information is carried by small number of modes. In fact, in an example we provide in a later section, the resultant density function does not change meaningfully once the number of $\xi$-mode reaches $\sim 30$. This feature combined with the decoupled dynamics of characteristic function is expected to weaken the curse of dimensionality significantly, at least compared to typical PDE approaches.

Analytical calculation is also possible. Since the dynamics is linear, one easily obtains the following results:

\section{Zeroth order}

$$
\rho_{t}^{[0]}(\psi(\xi))=e^{\int_{0}^{t} A_{s}^{[0]}(\xi) d s} \rho_{0}(\psi(\xi))
$$




\section{First order}

$$
\begin{aligned}
\rho_{t}^{[1]}(\psi(\xi))=\int_{0}^{t} e^{\int_{s}^{t} A_{u}^{[0]}(\xi) d u}\left\{A_{s}^{[1]}\left(\xi, D_{\xi}\right) \rho_{s}^{[0]}(\psi(\xi)) d s\right. \\
\\
\left.\quad+\left(H_{s}^{\top}\left(D_{\xi}\right)+i \xi^{\top} \gamma_{s}\left(D_{\xi}\right)\right) \rho_{s}^{[0]}(\psi(\xi)) d Y_{s}\right\}
\end{aligned}
$$

Higher order $(j \geq 2)$

Using the lower order results, an arbitrary order of the expansion can be expressed recursively as

$$
\begin{gathered}
\rho_{t}^{[j]}(\psi(\xi))=\int_{0}^{t} e^{\int_{s}^{t} A_{u}^{[0]}(\xi) d u}\left\{\left(A_{s}^{[1]}\left(\xi, D_{\xi}\right) \rho_{s}^{[j-1]}(\psi(\xi))+A_{s}^{[2]}\left(\xi, D_{\xi}\right) \rho_{s}^{[j-2]}(\psi(\xi))\right) d s\right. \\
\left.+\left(H_{s}^{\top}\left(D_{\xi}\right)+i \xi^{\top} \gamma_{s}\left(D_{\xi}\right)\right) \rho_{s}^{[j-1]}(\psi(\xi)) d Y_{s}\right\}
\end{gathered}
$$

Rigorous mathematical proofs of the validity and convergence of the above asymptotic expansion when taking $\epsilon \downarrow 0$ are beyond the scope of the current work. However, for a given observation path $Y$, it is likely to be proved by a similar line of arguments for the asymptotic expansion in position space without filtering problem given in Takahashi (1999), which is based on the results of Yoshida (1992a, 1992b, 1997) and Ikeda and Watanabe (1989). In the next section, we explain the inversion method to obtain the density function.

\subsection{Density Formula}

In this section, we provide a strategy to obtain an analytical expression of the filtered density. Although this is not necessary if one is only interested in numerical implementation with discrete Fourier transformation, the analytical expression can be quite useful for various applications in finance. In particular, a model calibration and quick response to a client request of price indication require very fast evaluation.

Let us consider the inverse Fourier transformation of $\rho_{t}^{(\epsilon)}(\psi(\xi))$ :

$$
\phi_{t}^{(\epsilon)}(z)=\frac{1}{(2 \pi)^{n}} \int_{\mathbb{R}^{n}} e^{-i \xi^{\top} z} \rho_{t}^{(\epsilon)}(\psi(\xi)) d^{n} \xi
$$

This corresponds to the unnormalized conditional probability density of the signal $X_{t}^{(\epsilon)}$ given the observation path of $Y$ (See the discussion in Sec 2.2.). The desired normalized conditional probability density of the signal is then given by

$$
\bar{\phi}_{t}^{(\epsilon)}(z)=\frac{1}{c_{t}^{(\epsilon)}} \phi_{t}^{(\epsilon)}(z)
$$

where $c_{t}^{(\epsilon)}$ is a normalization factor defined as

$$
\left(c_{t}^{(\epsilon)}\right)^{-1}=\int_{\mathbb{R}^{n}} \phi_{t}^{(\epsilon)}(z) d^{n} z
$$

Thus, for applications, it suffices to calculate the expression of $\phi_{t}^{(\epsilon)}(z)$. 


\subsubsection{Gaussian distribution for $X_{0}$}

For simplicity, let us first consider the case where $X_{0}$ is distributed by a Gaussian law $\mathcal{N}\left(x_{0} ; \Sigma_{0}\right)$ with mean $x_{0}$ and the covariance $\Sigma_{0}$ of a symmetric positive definite matrix. In this case, we have

$$
\begin{aligned}
\rho_{0}(\psi(\xi)) & =\int_{\mathbb{R}^{n}} e^{i \xi^{\top} x} n\left[x ; x_{0}, \Sigma_{0}\right] d^{n} x \\
& =\frac{1}{\sqrt{(2 \pi)^{n}\left|\Sigma_{0}\right|}} \int_{\mathbb{R}^{n}} e^{i \xi^{\top} x} \exp \left(-\frac{1}{2}\left(x-x_{0}\right)^{\top} \Sigma_{0}^{-1}\left(x-x_{0}\right)\right) d^{n} x
\end{aligned}
$$

where $n\left[x ; x_{0}, \Sigma_{0}\right]$ is the probability density function for a random variable with Gaussian low of $\mathcal{N}\left(x_{0} ; \Sigma_{0}\right)$, and $\left|\Sigma_{0}\right|$ denotes the determinant of $\Sigma_{0}$. The evaluation can be done easily by considering the variable change from $x$ to $\eta$ given by

$$
x=x_{0}+P_{0} \eta
$$

with a matrix $P_{0}$ satisfying

$$
\Sigma_{0}=P_{0} P_{0}^{\top}
$$

Integration in terms of $\eta$ leads to

$$
\rho_{0}(\psi(\xi))=\exp \left(i \xi^{\top} x_{0}-\frac{1}{2} \xi^{\top} \Sigma_{0} \xi\right)
$$

Then, from (11) of the previous section, we have

$$
\rho_{t}^{[0]}(\psi(\xi))=\exp \left(i \xi^{\top} x_{t}-\frac{1}{2} \xi^{\top} \Sigma_{t} \xi\right)
$$

where

$$
\begin{aligned}
& x_{t}=x_{0}+\int_{0}^{t} f_{s} d s \\
& \Sigma_{t}=\Sigma_{0}+\int_{0}^{t} \nu_{s} \nu_{s}^{\top} d s .
\end{aligned}
$$

Thus, it is clear that $X_{t}^{[0]}$ has a Gaussian distribution $\mathcal{N}\left(x_{t} ; \Sigma_{t}\right)$. If the initial position of $X_{0}$ is exactly known as $X_{0}=x_{0}$, then one clearly has

$$
\rho_{0}(\psi(\xi))=e^{i \xi^{\top} x_{0}}
$$

and hence one can simply insert $\Sigma_{0}=0$ in (14).

By the property of the exponential form and (12), one can check that $\rho_{t}^{[1]}(\psi(\xi))$ is given by

$$
\rho_{t}^{[1]}(\psi(\xi))=\rho_{t}^{[0]}(\psi(\xi))\left(\int_{0}^{t} a_{s}(\xi) d s+b_{s}(\xi) d Y_{s}\right)
$$

with polynomial functions $a_{s}(\xi) \in \mathbb{R}$ and $b_{s}(\xi) \in \mathbb{R}^{1 \times m}$ of $\xi$

$$
\begin{aligned}
& a_{s}(\xi)=\rho_{s}^{[0]}(\psi(\xi))^{-1} A_{s}^{(1)}\left(\xi, D_{\xi}\right) \rho_{s}^{[0]}(\psi(\xi)) \\
& b_{s}(\xi)=\rho_{s}^{[0]}(\psi(\xi))^{-1}\left(H_{s}\left(D_{\xi}\right)^{\top}+i \xi^{\top} \gamma_{s}\left(D_{\xi}\right)\right) \rho_{s}^{[0]}(\psi(\xi))
\end{aligned}
$$

which can be expressed by Hermite polynomials in general. 
Thus, the first order correction to the unnormalized conditional density can be expressed as

$$
\begin{aligned}
\phi_{t}^{[1]}(z) & :=\frac{1}{(2 \pi)^{n}} \int_{\mathbb{R}^{n}} e^{-i \xi^{\top} z} \rho_{t}^{[1]}(\psi(\xi)) d^{n} \xi \\
& =\left(\int_{0}^{t} a_{s}\left(D_{z}\right) d s+b_{s}\left(D_{z}\right)^{\top} d Y_{s}\right) n\left[z ; x_{t}, \Sigma_{t}\right]
\end{aligned}
$$

Here,

$$
D_{z}:=i \frac{\partial}{\partial z}
$$

and $a_{s}\left(D_{z}\right), b_{s}\left(D_{z}\right)$ denote the derivative operator of $z$ obtained by replacing each $\xi$ in the functions by $D_{z}$.

Repeating the same arguments, one can see that $\rho_{t}^{[j]}(\psi(\xi))$ can be given, as a generalization of (15), by

$$
\begin{aligned}
\rho_{t}^{[j]}(\psi(\xi))=\rho_{t}^{[0]}(\psi(\xi)) \int_{0}^{t} & \int_{0}^{s_{j}} \cdots \int_{0}^{s_{2}}\left(\Gamma_{s_{1}, \cdots, s_{j}}(\xi) d s_{1} d s_{2} \cdots d s_{j}\right. \\
& +\Gamma_{y_{1}, s_{2}, \cdots, s_{j}}(\xi) d Y_{s_{1}} d s_{2} \cdots d s_{j} \\
\cdots & \\
& \left.+\Gamma_{y_{1}, y_{2}, \cdots, y_{j}}(\xi) d Y_{s_{1}} d Y_{s_{2}} \cdots d Y_{s_{j}}\right)+\cdots
\end{aligned}
$$

with certain polynomial functions $\left\{\Gamma_{s_{1}, \cdots, s_{j}}(\xi), \cdots, \Gamma_{y_{1}, \cdots, y_{j}}(\xi)\right\}$ of $\xi$ with appropriate dimensions. The $\{\cdots\}$ denotes the term with integration order of $(<j)$, which stems from the existence of the $\epsilon$-second order operator $A^{[2]}$. Then, as a generalization of (16), the $j$-th order term of the unnormalized conditional density is also given by the correction to the Gaussian distribution:

$$
\begin{aligned}
\phi_{t}^{[j]}(z):= & \frac{1}{(2 \pi)^{n}} \int_{\mathbb{R}^{n}} e^{-i \xi^{\top} z} \rho_{t}^{[j]}(\psi(\xi)) d^{n} \xi \\
= & \int_{0}^{t} \int_{0}^{s_{j}} \cdots \int_{0}^{s_{2}}\left(\Gamma_{s_{1}, \cdots, s_{j}}\left(D_{z}\right) d s_{1} d s_{2} \cdots d s_{j}\right. \\
& +\Gamma_{y_{1}, s_{2}, \cdots, s_{j}}\left(D_{z}\right) d Y_{s_{1}} d s_{2} \cdots d s_{j} \\
& \quad \cdots \\
& \left.+\Gamma_{y_{1}, y_{2}, \cdots, y_{j}}\left(D_{z}\right) d Y_{s_{1}} d Y_{s_{2}} \cdots d Y_{s_{j}}\right) n\left[z ; x_{t}, \Sigma_{t}\right]+\cdots
\end{aligned}
$$

In the case of no (or trivial) observation, one can get the asymptotic expansion of unconditional probability density by putting $d Y$ terms zero.

\subsubsection{Non-Gaussian distribution for $X_{0}$}

Even when the initial distribution is not exactly Gaussian, if one can approximate it by the form

$$
\phi_{0}(z)=(\text { some polynomial function of } \mathrm{z}) \times n\left[z ; x_{0}, \Sigma_{0}\right],
$$

then the properties of the inverse transformation given in the previous section still hold in almost the same way. This is, for example, the case when one approximates the initial distribution by Gram-Charlier expansions. In the case when (19) holds, one can still write $\rho_{t}^{[0]}$ in the form

$$
\rho_{t}^{[0]}(\psi(\xi))=(\text { some polynomial function of } \xi) \times \exp \left(i \xi^{\top} x_{t}-\frac{1}{2} \xi^{\top} \Sigma_{t} \xi\right)
$$

and it only changes the functions $\{\Gamma\}$ in (17) and (18). 


\section{A Direct Application to Kushner-Stratonovich Equation}

We can also apply the technique to the Kushner-Stratonovich (KS) equation that describes the dynamics of the normalized conditional density of $\pi_{t}$ instead of $\rho_{t}$. Although it suffices to work on the simpler Zakai equation in filtering problems, we directly treat KS equation here to demonstrate the fact that the asymptotic expansion can also be applied to measure-valued non-linear systems. For the setup given in Sec 2.1, the Kushner-Stratonovich equation is given by

$$
d \pi_{t}(\varphi)=\pi_{t}\left(A_{t} \varphi\right) d t+\left\{\pi_{t}\left(\left(h_{t}+B_{t}\right)^{\top} \varphi\right)-\pi_{t}\left(h_{t}^{\top}\right) \pi_{t}(\varphi)\right\}\left(d Y_{t}-\pi_{t}\left(h_{t}\right) d t\right)
$$

with a given initial value $\pi_{0}(\varphi)$. This is clearly a nonlinear equation for the measurevalued process $\pi_{t}$. See a textbook Bain and Crisan (2008) for details of the derivation.

Let us now introduce the same perturbed system as in Sec 3.1. Then, one obtains the $\mathrm{KS}$ equation for $\psi(\xi, \cdot)$ as

$$
\begin{aligned}
& d \pi_{t}^{(\epsilon)}(\psi(\xi))=\pi_{t}^{(\epsilon)}\left(A_{t}^{(\epsilon)} \psi(\xi)\right) d t \\
& \quad+\epsilon\left\{\pi_{t}^{(\epsilon)}\left(\left(H_{t}+B_{t}\right)^{\top} \psi(\xi)\right)-\pi_{t}^{(\epsilon)}\left(H_{t}^{\top}\right) \pi_{t}^{(\epsilon)}(\psi(\xi))\right\}\left(d Y_{t}-\epsilon \pi_{t}^{(\epsilon)}\left(H_{t}\right) d t\right)
\end{aligned}
$$

By the same polynomial-function approximations, one can rewrite it as

$$
\begin{aligned}
& d \pi_{t}^{(\epsilon)}(\psi(\xi))=\left(A_{t}^{[0]}(\xi)+\epsilon A_{t}^{[1]}\left(\xi, D_{\xi}\right)+\epsilon^{2} A_{t}^{[2]}\left(\xi, D_{\xi}\right)\right) \pi_{t}^{(\epsilon)}(\psi(\xi)) d t \\
& \quad+\epsilon\left\{\left(H_{t}^{\top}\left(D_{\xi}\right)+i \xi^{\top} \gamma_{t}\left(D_{\xi}\right)\right) \pi_{t}^{(\epsilon)}(\psi(\xi))-\pi_{t}^{(\epsilon)}\left(H_{t}^{\top}\right) \pi_{t}^{(\epsilon)}(\psi(\xi))\right\}\left(d Y_{t}-\epsilon \pi_{t}^{(\epsilon)}\left(H_{t}\right) d t\right) .
\end{aligned}
$$

As before, we try to expand the solution as

$$
\pi_{t}^{(\epsilon)}(\psi(\xi))=\pi_{t}^{[0]}(\psi(\xi))+\epsilon \pi_{t}^{[1]}(\psi(\xi))+\epsilon^{2} \pi_{t}^{[2]}(\psi(\xi))+\cdots
$$

with the definition

$$
\pi_{t}^{[j]}(\psi(\xi)):=\left.\frac{1}{j !} \frac{\partial^{j}}{\partial \epsilon^{j}} \pi_{t}^{(\epsilon)}(\psi(\xi))\right|_{\epsilon=0} .
$$

Note that there appears $\pi_{t}^{(\epsilon)}\left(H_{t}\right)$ in (20). This term does no harm since an arbitrary order $j$ of asymptotic expansion, we need $\pi_{t}^{[i]}\left(H_{t}\right)$ only for $i=0,1, \cdots, j-1$ due to the additional $\epsilon$-factor. Thus, at the calculation of $j$-th order expansion, one can use

$$
\pi_{t}^{[i]}\left(H_{t}\right)=\left.H_{t}\left(D_{\xi}\right) \pi_{t}^{[i]}(\psi(\xi))\right|_{\xi=0}
$$

where $\pi_{t}^{[i]}(\psi(\xi))$ are already known for $i \leq j-1$.

Let us give the first few orders of expansions for the KS equation:

\section{Zeroth order}

$$
d \pi_{t}^{[0]}(\psi(\xi))=A_{t}^{[0]}(\xi) \pi_{t}^{[0]}(\psi(\xi)) d t
$$

with initial value $\pi_{0}^{[0]}(\psi(\xi))=\pi_{0}(\psi(\xi))$.

\section{First oder}

$$
\begin{aligned}
& d \pi_{t}^{[1]}(\psi(\xi))=A_{t}^{[0]}(\xi) \pi_{t}^{[1]}(\psi(\xi)) d t \\
& \quad+\left\{\left(H_{t}^{\top}\left(D_{\xi}\right)+i \xi^{\top} \gamma_{t}\left(D_{\xi}\right)\right) \pi_{t}^{[0]}(\psi(\xi))-\pi_{t}^{[0]}\left(H_{t}^{\top}\right) \pi_{t}^{[0]}(\psi(\xi))\right\} d Y_{t}
\end{aligned}
$$


with $\pi_{0}^{[1]}(\psi(\xi))=0$.

\section{Second order}

$$
\begin{aligned}
d \pi_{t}^{[2]} & (\psi(\xi))=A_{t}^{[0]}\left(\xi, D_{\xi}\right) \pi_{t}^{[2]}(\psi(\xi)) d t \\
& +\left\{A_{t}^{[1]}\left(\xi, D_{\xi}\right) \pi_{t}^{[1]}(\psi(\xi))+A_{t}^{[2]}\left(\xi, D_{\xi}\right) \pi_{t}^{[0]}(\psi(\xi))\right. \\
& \left.-\left(H_{t}^{\top}\left(D_{\xi}\right)+i \xi^{\top} \gamma_{t}\left(D_{\xi}\right)-\pi_{t}^{[0]}\left(H_{t}^{\top}\right)\right) \pi_{t}^{[0]}(\psi(\xi)) \pi_{t}^{[0]}\left(H_{t}\right)\right\} d t \\
& +\left\{\left(H_{t}^{\top}\left(D_{\xi}\right)+i \xi^{\top} \gamma_{t}\left(D_{\xi}\right)-\pi_{t}^{[0]}\left(H_{t}^{\top}\right)\right) \pi_{t}^{[1]}(\psi(\xi))-\pi_{t}^{[1]}\left(H_{t}^{\top}\right) \pi_{t}^{[0]}(\psi(\xi))\right\} d Y_{t}
\end{aligned}
$$

with initial condition $\pi_{t}^{[2]}(\psi(\xi))=0$.

Although the system of ODEs does not keep the same structure as the unnormalized distribution $\rho$, it is clear that one can still perform the perturbation order by order. Furthermore, from the discussion given in the next section, it is not always necessary to derive higher order asymptotic expansion for accurate estimation.

\section{Substepping Method for Asymptotic Expansion}

It is obvious by construction that the accuracy of approximation deteriorates once the cumulative contributions from perturbation terms

$$
\epsilon F, \quad \epsilon \sigma, \quad \epsilon \gamma, \quad \epsilon H
$$

become significant. This is a common problem of asymptotic expansion methods for various applications. In particular for the filtering problems, requiring small perturbation terms seems rather restrictive since it indicates that one can treat only noisy observations (i.e. small $h$ ). In financial applications of the position-space asymptotic expansion, it is known that one needs higher order approximation to reach enough accuracy for practical use in long-dated or high-volatility environments. There exist many efforts to obtain higher order corrections systematically to tackle these problems. See, for example, Takahashi and Takehara (2007), Takahashi et al. (2010), and $\mathrm{Li}$ (2010) for recent developments in this direction.

Let us consider the problem in the momentum-space approach. In Theorem 1, we have seen that the equation (9) determines the correction terms with a given initial condition $\rho_{0}(\psi(\xi))$. Although higher order calculation is straightforward, there exists a simpler and more efficient way to improve the approximation. An obvious but important feature of (9) is that the recursion can be started from an arbitrary initial distribution $\rho_{0}(\psi(\xi))$. Since asymptotic expansion generally works very well for short maturities, the above feature naturally leads to the following substepping idea for asymptotic expansion.

(1) Create an appropriate time grid $\left\{0=T_{0}<T_{1}<\cdots<T_{N}=t\right\}$ in such a way that the asymptotic expansion converges well within each sub-period $\left[T_{i-1}, T_{i}\right]$.

(2) Solve (9) of each $\xi$ for $s \in\left[T_{0}, T_{1}\right]$ up to the $k$-th order of the asymptotic expansion. This can be the second (or even the first) order if the stepping size is small enough.

(3) Update the initial condition for the next period $\left[T_{1}, T_{2}\right]$ by setting

$$
\rho_{T_{1}}^{[0]}(\psi(\xi)) \leftarrow\left(\sum_{j=0}^{k} \epsilon^{j} \rho_{T_{1}}^{[j]}(\psi(\xi))\right) \text { obtained in step(2) }
$$


(4) Solve (9) for $\left[T_{1}, T_{2}\right]$ with the updated initial condition.

(5) Repeat the procedures till the final period to obtain $\rho_{T_{N}}^{[j]}(\psi(\xi))$.

Although performing the above method analytically by hand is quite laborious due to a large number of derivative operations, one can do it quite efficiently in numerical implementations. This is because amount of procedures required for $d t$ and $d Y$ integration does not change in the above operations. In fact, one obtains accurate results faster by performing finer substepping with lower-order approximation than performing higher-order approximation without substepping.

The substepping method may be very useful for general measure-valued nonlinear equations. Although it is tedious to obtain the asymptotic expansion for complicated dynamics, it is definitely possible for the first few orders as we have demonstrated by using the Kushner-Stratonovich equation. If the approximation works well, at least within a very short period, the above numerical procedures will extend the effective region for the asymptotic expansion. If it is applied to a standard unconditional characteristic function, it should also offer an efficient option pricing method, particularly for long-dated and high-volatile setups.

\section{Examples}

\subsection{Analytical Application to CIR Process}

Let us first consider the approximation of one-dimensional CIR process with no filtering issue, which helps to obtain a concrete image how analytical procedures work. We study

$$
d X_{t}=\theta\left(\mu-X_{t}\right) d t+\sigma \sqrt{X_{t}} d V_{t}
$$

with $X_{0}=\mu$. All the parameters $\theta, \mu$ and $\sigma$ are positive constants satisfying $2 \theta \mu>$ $\sigma^{2}$. Then, the probability density of $X_{t}$ is known to have a non-central chi-squared distribution.

For asymptotic expansion, we treat it as the following perturbed system: 1

$$
d X_{t}^{(\epsilon)}=\epsilon \theta F\left(X_{t}^{(\epsilon)}\right) d t+\sigma\left(\sqrt{\mu}+\epsilon R\left(X_{t}^{(\epsilon)}\right)\right) d V_{t}
$$

with $X_{0}^{(\epsilon)}=\mu$. Here, we have defined

$$
\begin{aligned}
& F(x)=\mu-x \\
& R(x)=\text { Taylor expansion at }(x=\mu) \text { of }(\sqrt{x}-\sqrt{\mu}) .
\end{aligned}
$$

In this example, we are going to adopt the 3rd-order expansion for $R(x)$. Note that, Taylor expansion provides a good polynomial approximation only when the process $X$ resides near $\mu$. If volatility is very high, it may be better to perform a different method, such as the minimization of the least square difference for appropriate range. We shall see some examples in the next section. Systematic strategy for determining the optimal choice of polynomial function remains as an important future work.

Then, the infinitesimal generator is given by

$$
A^{(\epsilon)}=\epsilon \theta F(x) \frac{\partial}{\partial x}+\frac{1}{2} \sigma^{2}(\sqrt{\mu}+\epsilon R(x))^{2} \frac{\partial^{2}}{\partial x^{2}}
$$

\footnotetext{
1 One needs to put $\epsilon=1$ at the end for the comparison to the original model.
} 
and hence

$$
\begin{aligned}
& A^{[0]}(\xi)=-\frac{1}{2} \xi^{2} \sigma^{2} \mu \\
& A^{[1]}\left(\xi, D_{\xi}\right)=i \xi \theta F\left(D_{\xi}\right)-\xi^{2} \sigma^{2} \sqrt{\mu} R\left(D_{\xi}\right) \\
& A^{[2]}\left(\xi, D_{\xi}\right)=-\frac{1}{2} \xi^{2} \sigma^{2} R^{2}\left(D_{\xi}\right) .
\end{aligned}
$$

From Corollary 1, analytical calculation can be performed as follows:

\subsubsection{Zeroth order}

We have

$$
d \rho_{t}^{[0]}(\psi(\xi))=A^{[0]}(\xi) \rho_{t}^{[0]}(\psi(\xi)) d t
$$

with $\rho_{0}^{[0]}(\psi(\xi))=e^{i \xi \mu}$. Thus, it gives

$$
\rho_{t}^{[0]}(\psi(\xi))=\exp \left(i \xi \mu-\frac{1}{2} \xi^{2} \sigma^{2} \mu t\right) .
$$

Then, one obtains the zeroth order density function as

$$
\phi_{t}^{[0]}(z)=\frac{1}{\sqrt{2 \pi \Sigma_{t}}} \exp \left(-\frac{(\mu-z)^{2}}{2 \Sigma_{t}}\right)
$$

with the definition of $\Sigma_{t}=\mu \sigma^{2} t$.

\subsubsection{First order}

The first order correction is given by

$$
\rho_{t}^{[1]}(\psi(\xi))=\int_{0}^{t} e^{(t-s) A^{[0]}(\xi)} A^{[1]}\left(\xi, D_{\xi}\right) \rho_{s}^{[0]}(\psi(\xi)) d s .
$$

By straightforward differential operations lead to

$$
\begin{aligned}
\left(\rho_{s}^{[0]}(\psi(\xi))\right)^{-1} A^{[1]}\left(\xi, D_{\xi}\right) \rho_{s}^{[0]}(\psi(\xi))= & \frac{1}{8} s \sigma^{2}\left(8 \theta \mu+\sigma^{2}\right) \xi^{2}-\frac{1}{16} i s \sigma^{4}\left(8 \mu+3 s \sigma^{2}\right) \xi^{3} \\
& -\frac{1}{8} s^{2} \mu \sigma^{6} \xi^{4}+\frac{1}{16} i s^{3} \mu \sigma^{8} \xi^{5} .
\end{aligned}
$$

Then, following the procedures of Sec. 3.3, one obtains

$$
\rho_{t}^{[1]}(\psi(\xi))=\rho_{t}^{[0]}\left(a_{2} \xi^{2}+a_{3} \xi^{3}+a_{4} \xi^{4}+a_{5} \xi^{5}\right)
$$

and also

$$
\begin{aligned}
\phi_{t}^{[1]}(z) & =\frac{1}{2 \pi} \int_{\mathbb{R}} e^{-i \xi z} \rho_{t}^{[1]}(\psi(\xi)) d \xi \\
& =\left(-a_{2} \frac{\partial}{\partial z^{2}}-i a_{3} \frac{\partial^{3}}{\partial z^{3}}+a_{4} \frac{\partial^{4}}{\partial z^{4}}+i a_{5} \frac{\partial^{5}}{\partial z^{5}}\right) \phi_{t}^{[0]}(z)
\end{aligned}
$$

with the coefficients defined by

$$
\begin{aligned}
& a_{2}=\frac{1}{16} t^{2} \sigma^{2}\left(8 \theta \mu+\sigma^{2}\right), \quad a_{3}=-\frac{1}{16} i t^{2} \sigma^{4}\left(4 \mu+t \sigma^{2}\right) \\
& a_{4}=-\frac{1}{24} t^{3} \mu \sigma^{6}, \quad a_{5}=\frac{1}{64} i t^{4} \mu \sigma^{8} .
\end{aligned}
$$


Higher order expressions follow similarly with the help of analytical software if necessary. In Figs. 1 and 2 we have given numerical results up to $\epsilon$-3rd order asymptotic expansions without substepping compared with the exact non-central chi-squared distribution. When volatility is large, there appears sizable deviation from the correct distribution for small $x$. This is understandable because Taylor expansion near the origin is not accurate. Except a neighbor of the origin and $(x<0)$, one can see that our approximation reproduces the desired density function well.

\subsection{A Numerical Application to Beneš Filter}

Next, as the second example, we study the Beneš filter [Beneš (1981)], where the drift of the signal process is nonlinear. This is a special case for which there exists an exact solution in the class of non-Gaussian filtering problems, thus it is quite useful for testing the current method. In the class of Beneš filter, we choose a following one-dimensional example:

$$
\begin{aligned}
& d X_{t}=f\left(X_{t}\right) d t+\sigma d V_{t} \\
& d Y_{t}=\left(h_{1} X_{t}+h_{2}\right) d t+d W_{t}
\end{aligned}
$$

with $X_{0}=Y_{0}=0$, where $f(x)$ is given by

$$
f(x)=a \sigma \tanh \left(a \frac{x}{\sigma}\right) .
$$

Here, $a, \sigma, h_{1}$ and $h_{2}$ are all constants. In this case, the exact filtered density of $X_{t}$ is given by

$$
\begin{aligned}
& \pi_{t}^{\text {exact }}(z) \\
& =\frac{1}{n_{t}} \cosh \left(a \frac{z}{\sigma}\right) \exp \left(-\frac{h_{1}}{2 \sigma} \operatorname{coth}\left(t h_{1} \sigma\right) z^{2}\right. \\
& \left.\quad+\left(h_{1} \int_{0}^{t} \frac{\sinh \left(s h_{1} \sigma\right)}{\sinh \left(h_{1} \sigma\right)} d Y_{s}+\frac{h_{2}}{\sigma \sinh \left(t h_{1} \sigma\right)}-\frac{h_{2}}{\sigma} \operatorname{coth}\left(t h_{1} \sigma\right)\right) z\right)
\end{aligned}
$$

where $n_{t}$ is the normalization factor to guarantee

$$
\int_{\mathbb{R}} \pi_{t}^{\text {exact }}(z) d z=1
$$

For this problem, we setup the following perturbed approximation: 2

$$
\begin{aligned}
& d X_{t}^{(\epsilon)}=\epsilon F\left(X_{t}^{(\epsilon)}\right) d t+\sigma d V_{t} \\
& d Y_{t}=\epsilon H\left(X_{t}^{(\epsilon)}\right) d t+d W_{t}
\end{aligned}
$$

with $X_{0}^{(\epsilon)}=Y_{0}=0$. Here, we use

$$
\begin{aligned}
& H(x)=h_{1} x+h_{2} \\
& F(x)=\text { (polynomial approximation of) } f(x) .
\end{aligned}
$$

We explain the details of polynomial approximation later.

The infinitesimal generator contains only up to $\epsilon$-first order term. We have

$$
A^{(\epsilon)}=\epsilon F(x) \frac{\partial}{\partial x}+\frac{1}{2} \sigma^{2} \frac{\partial^{2}}{\partial x^{2}}
$$

\footnotetext{
${ }^{2}$ As before, one needs to put $\epsilon=1$ at the end for the comparison to the original model.
} 
and

$$
A^{[0]}(\xi)=-\frac{1}{2} \xi^{2} \sigma^{2}, \quad A^{[1]}\left(\xi, D_{\xi}\right)=i \xi F\left(D_{\xi}\right) .
$$

From Theorem 1, one needs to solve the recursion for $(j \geq 1)$

$\rho_{t}^{[j]}(\psi(\xi))=\int_{0}^{t} e^{-\frac{1}{2}(t-s) \xi^{2} \sigma^{2}}\left\{i \xi F\left(D_{\xi}\right) \rho_{s}^{[j-1]}(\psi(\xi)) d s+\left(h_{1} D_{\xi}+h_{2}\right) \rho_{s}^{[j-1]}(\psi(\xi)) d Y_{s}\right\}$

starting from the zeroth order solution:

$$
\rho_{t}^{[0]}(\psi(\xi))=\exp \left(-\frac{1}{2} t \xi^{2} \sigma^{2}\right)
$$

\subsubsection{Polynomial-function approximation}

Here, we discuss how to obtain the polynomial approximation $F(x)$ for the nonlinear drift of the signal:

$$
f(x)=a \sigma \tanh (a x / \sigma) .
$$

Due to the normalization by $\sigma$, we can roughly expect

$$
\left|\frac{X_{t}}{\sigma}\right| \lesssim 1
$$

for $t \in[0,1]$. This implies that Taylor expansion around $x=0$ is a natural candidate for $F(x)$ when $|a| \lesssim 1$. When $|a| \gtrsim 1$, the two plateaus of $f(x)$ start to play an important role in the range (28). Unfortunately, however, Taylor expansion does not reproduce the plateaus but strongly diverging behavior within the range (28) instead, which destabilizes the numerical result. Thus, we take $[-5 \sigma, 5 \sigma]$ range with a step size of $0.2 \sigma$, and carry out least-square method (LSM) to fit a 11-dimensional odd function for $F(x)$. We also adopt the weight function $g(x)$ defined as

$$
g(x)=\exp \left(-w \frac{x^{2}}{2 \sigma^{2}}\right)
$$

with various factor $w$. Here, $w=0$ corresponds to a pure LSM in the $5 \sigma$ range and the polynomial function well recovers the two plateaus of $f(x)$ in a wide range, while it has a relatively poor fit around the origin. On the other hand, higher $w$ gives finer fit and hence finer description of the density near $x=0$. In this case, however, if one continues to increase $w$ it starts to destabilize the numerical result as in the case for Taylor expansion. Thus, we need to take a balance of this trade-off, especially when $|a|>1$.

\subsubsection{Numerical Results}

In the following numerical examples, we take $t=1$ as the maturity and use 1,000 steps to create the sample observation (and signal) path. We then integrate (27) with the same time step $d t=10^{-3}$ for a given path of $Y$. For differentiation, we use a standard finite difference method. Finally, a discrete Fourier transformation is used to obtain the density function.

In the first numerical example given in Fig. 3, we have used a set of parameters $\left\{a=0.8, \sigma=0.5, h_{1}=0.8, h_{2}=0.5\right\}$, and a sample path of $Y$ given in the top graph. We have used $w=2.0$ for getting coefficients of polynomial function $F(x)$. The middle graph for the conditional density functions contains the exact one denoted by 
a red line labeled as "Benes", estimated conditional densities from (0th, 3rd, 20th)order asymptotic expansion without substepping method, and those from 1st order expansion with substepping method of $(100,1000)$ sub-periods 3 . One can clearly see the benefit of substepping method explained in Sec. 5 Although there is no clear improvement from 3rd to 20th order approximation, the substepping method with small sub-period provides almost exact fit to the true density function.

In Fig. 4, we have used $h_{1}=10.0$, which is an example of small-noise observation. Since $a=0.5$ is relatively small, polynomial approximation for $f(x)$ is quite accurate $(w=2.0)$. The calculation has been performed with substepping method of $(100,125$, $200,1,000)$ sub-periods. Fine substepping gives almost exact density even in this case. In particular, the significant reduction of the variance of the conditional density due to high quality information provided by the observation process is well reproduced by the repeated application of asymptotic expansion. In this example, approximation without substepping is too unstable and gives only meaningless numerical results. As suggested in Sec. 5, the size of perturbation terms itself does not seem to be a relevant problem for asymptotic expansion as long as we have accurate enough polynomial function approximation and the substepping method.

When one increases $a, f(x)$ becomes like a step function and makes it difficult to achieve accurate polynomial approximation for the relevant range. Here, the choice of LSM weight $w$ starts to affect the estimated density. In Fig. 5] we have studied the case of $a=1.5$ with several choice of $w$. Here, all the calculations were done with substepping method of 1000 sub-periods. The estimated density is stable for $w=0.5 \sim 2.5$, but becomes unstable for higher $w$. Note that, the impacts of LSM weight are highly dependent on the order of the polynomial function. As is easily guessed, the change of estimated density is more significant when lower order polynomials are used. In Fig. 6, we have done similar analysis for an example of $a=2.0$, which reveals more clearly separated two peaks of the filtered density.

\section{Remark :}

In the above examples, we have used time-independent function for $F(x)$. However, when there exists a significant drift for the signal process, such as large $|a|$ in the above example, making the polynomial function time dependent is quite likely to improve the approximation. If we have the information about the evolution of the conditional mean, we can change the center of polynomial-function approximation to replicate the original nonlinear function more accurately in the relevant region. Initial guess can be obtained by extended Kalman-Bucy filter or by time independent $F(x)$ in the current method, for example.

\section{Concluding Remarks}

In the paper, we have developed an asymptotic expansion technique in momentum space. Fourier transformation combined with polynomial-function approximation gives a closed recursive system of ODEs as an asymptotic expansion for the unnormalized conditional characteristic function. Thanks to the simplicity of the ODE system, higher order calculation can be performed easily. It also allows an efficient implementation of substepping method of asymptotic expansion. As long as polynomial approximation of the nonlinear terms is accurate, the size of nonlinear terms ceases to be a big obstacle for obtaining an accurate estimation. Applications to more realistic multi-dimensional filtering problems as well as other (financial) problems, such

\footnotetext{
3 First order expansion is good enough for short period since the infinitesimal generator contains no 2 nd order term.
} 
as option pricing (with some unobservable parameters), are left for the future research.

Let us make a brief comment on the remaining problems and possible future directions of research to address these issues. As one can see, the method still suffers from the curse of dimensionality. However, encouragingly, there exist a large number of works to ameliorate the higher dimensional integration problem. See, for example, Griebel and Holtz (2010), Reisinger and Wittum (2007), Reisinger and Wissmann (2012) and Schröder et al. (2012). Especially, in Reisinger and Wissmann, the authors make use of the low effective dimensions of financial problems arising from a high correlation in the market. Although they have worked in restrictive model assumptions, they succeeded to approximate a high dimensional PDE by a series of low dimensional PDE. Applications and improvement of these techniques by combining the asymptotic expansion developed in this paper look quite interesting.

Since we can only use finite order polynomials in practice, the quality of the estimation highly depends on the accuracy of polynomial approximation. When one has nonlinear terms difficult to fit by polynomials, the idea of change-of-variable developed in Takahashi and Toda (2012) may be proved to be useful. Suppose, one defines a new process $\widetilde{X}$ by using some function $\Psi(\cdot)$ as

$$
\widetilde{X}_{t}=\Psi\left(X_{t}\right) \text {. }
$$

If $\widetilde{X}$ has drift and diffusion terms that are easier to be approximated by polynomial functions, one can get more accurate estimation of

$$
\widetilde{\rho}_{t}(\xi)=\mathbb{E}\left[e^{i \xi^{\top} \tilde{X}_{t}}\right]
$$

and hence also its density

$$
\widetilde{\phi}_{t}(z)=\frac{1}{(2 \pi)^{n}} \int_{\mathbb{R}^{n}} e^{-i \xi^{\top} z} \widetilde{\rho}_{t}(\xi) d^{n} \xi .
$$

Then, one can recover the density of the original $X_{t}$ by

$$
\phi_{t}(z)=\widetilde{\phi}(\Psi(z))|J(z)|
$$

where $|J|$ denotes the determinant of a Jacobian matrix with the elements of $\left(\partial \Psi_{i}(z) / \partial z_{j}\right)$. Thus some of the errors can be absorbed if there exists an appropriate choice of $\Psi$.

Another possible solution is to use Fourier series expansion directly for nonlinear functions in the infinitesimal generator and observation process 4 . Although it effectively increases the order of integration and hence slows down the calculation, some functions, such as step function, are known to allow accurate approximation by relatively a small number of terms. Suppose for example, some function $g$ has a Fourier expansion as

$$
g(x)=\sum_{n} \widetilde{g}_{n} e^{-i \xi_{n}^{\top} x}
$$

where $\left\{\xi_{n}\right\}$ is a series of discretized momentum, and $\left\{g_{n}\right\}$ is a set of corresponding coefficients. Then, one has

$$
\mathbb{E}\left[g(x) e^{i \xi_{m}^{\top} x}\right]=\sum_{n} \widetilde{g}_{n} \mathbb{E}\left[e^{i\left(\xi_{m}-\xi_{n}\right) x}\right]
$$

\footnotetext{
4 Note that, one have to resort to discrete Fourier transformation for numerical implementation anyway.
} 
Since all the nonlinear functions are included in the perturbation terms, one can write the dynamics of $\rho_{t}^{[j]}(\psi(\xi))$ in terms of $\left\{\rho_{t}^{[k]}\left(\psi\left(\xi_{n}\right)\right\}_{n}\right.$ with $k=\{j-1, j-2\}$. As a result, it is still linear for the highest expansion order and can be treated similarly as in Theorem 1. This technique may be crucial for financial applications where stiff payoff functions are common. Detailed studies of these points are also among our research topics in the future.

\section{Acknowledgment}

The author thanks to Akihiko Takahashi of University of Tokyo for informative discussions that help to understand the connections to the asymptotic expansion in the position space. This research is supported by CARF (Center for Advanced Research in Finance) and the global COE program "The research and training center for new development in mathematics."

\section{References}

1. Bain, A., Crisan, D., (2008). Fundamentals of Stochastic Filtering. New York. Springer.

2. Beneš, V.E., (1981). Exact finite-dimensional filters for certain diffusions with nonlinear drift. Stochastics, 5(1-2), 65-92.

3. Bucy, R. S., (1959). Optimum finite time filters for a special non-stationary class of inputs. Technical Report Internal Report B.B.D. 600, March 31, Johns Hopkins Applied Physics Laboratory.

4. Bungartz, H.-J., and Griebel, M., (2004). Sparse grids. Acta Numerica, 13, 1-123

5. Crisan, D. and Rozovskii, B., (2011). The Oxford Handbook of Nonlinear Filtering. New York. Oxford university press.

6. Frey, R. and Runggaldier, W., (2011). Nonlinear Filtering in Models for Interest-Rate and Credit Risk. An article in The Oxford Handbook of Nonlinear Filtering of Oxford University Press.

7. Griebel, M. and Holtz, M., (2010). Dimension-wise integration of high-dimensional functions with applications to finance. Journal of Complexity, 26(5), 455-489.

8. Ikeda, N. and Watanabe, S. (1989). Stochastic Differential Equations and Diffusion Processes. Second Edition, North-Holland/Kodansha, Tokyo.

9. Kalman, R.E., (1960). A new approach to liner filtering and prediction problems. Journal of Basic Engineering, 82, 35-45.

10. Kalman, R. E. and Bucy, R. S., (1961). New results in liner filtering and prediction theory. Journal of Basic Engineering, 83, 95-108

11. Li, C., (2010). Closed-form Expansion, Conditional Expectation, and Option Valuation. Guanghua School of Management, Peking University, Beijing, China.

12. Reisinger, C., and Wittum, G., (2007). Efficient hierarchical approximation of high-dimensional option pricing problems. SIAM Journal of Scientific Computing, 29, 440-458

13. Reisinger, C., and Wissmann, R., (2012). Numerical Valuation of Bermudan and Path-Dependent Interest Rate Derivatives via PDE Expansions. Working paper.

14. Schröder, P., Gerstner, T., and Wittum, G., (2012). Taylor-like ANOVA-expansion for high dimensional problems in finance. Working paper.

15. Takahashi, A., (1999). An Asymptotic Expansion Approach to Pricing Contingent Claims. AsiaPacific Financial Markets, Vol. 6, 115-151.

16. Takahashi, A. and Takehara, K., (2007). Pricing Currency Options with a Market Model of Interest Rates under Jump-Diffusion Stochastic Volatility Processes of Spot Exchange Rates. Asia-Pacific Financial Markets, Vol.14, pp. 69-121.

17. Takahashi, A., Takehara, K. and Toda. M., (2010). A General Computation Scheme for a HighOrder Asymptotic Expansion Method. CARF Working Paper, F-272, forthcoming in International Journal of Theoretical and Applied Finance.

18. Takahashi, A., Toda, M., (2012). Note on an Extension of an Asymptotic Expansion Scheme. CARF Working Paper F-286, available at http://www.carf.e.u-tokyo.ac.jp/workingpaper/

19. Yoshida, N.(1992a). Asymptotic Expansions for Statistics Related to Small Diffusions. Journal of Japan Statistical Society, Vol.22, 139-159.

20. Yoshida, N.(1992b). Asymptotic Expansions of Maximum Likelihood Estimators for Small Diffusions via the Theory of Malliavin-Watanabe. Probability Theory and Related Fields, Vol.92, 275-311.

21. Yoshida, N.(1997). Malliavin Calculus and Asymptotic Expansions for Martingales. Probability Theory and Related Fields, Vol.109, 301-342 


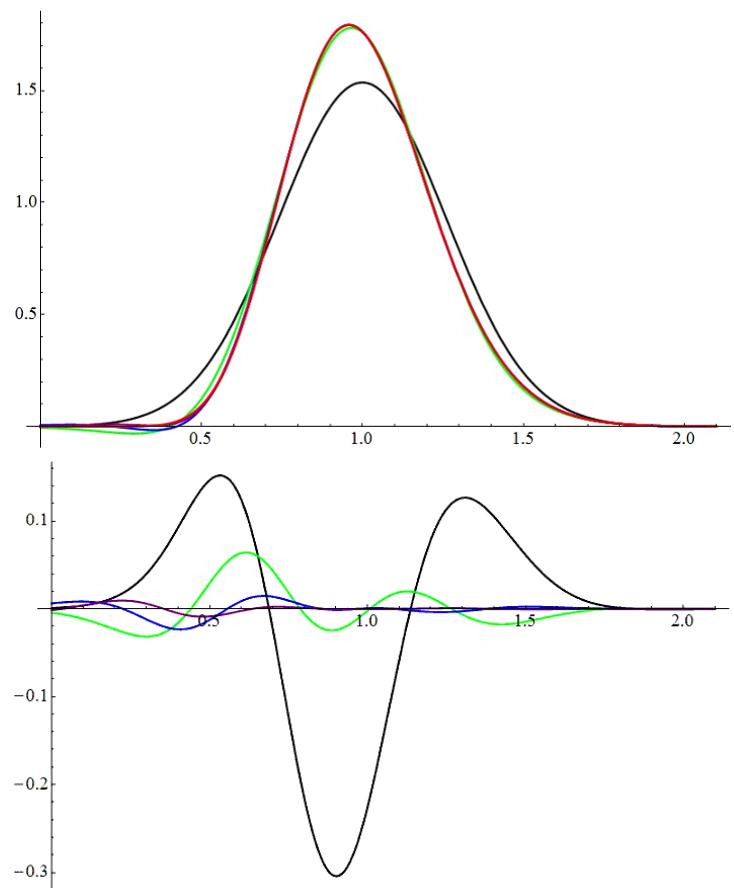

Fig. $1 t=3, \mu=1, \sigma=0.15, \theta=0.1$. (Black, Green, Blue, Purple) lines denote (0th, 1st, 2nd, 3rd) order approximation of asymptotic expansion, respectively. Red line denotes the exact density function given by a non-central chi-squared distribution. The second graph represents the difference from the exact density function.

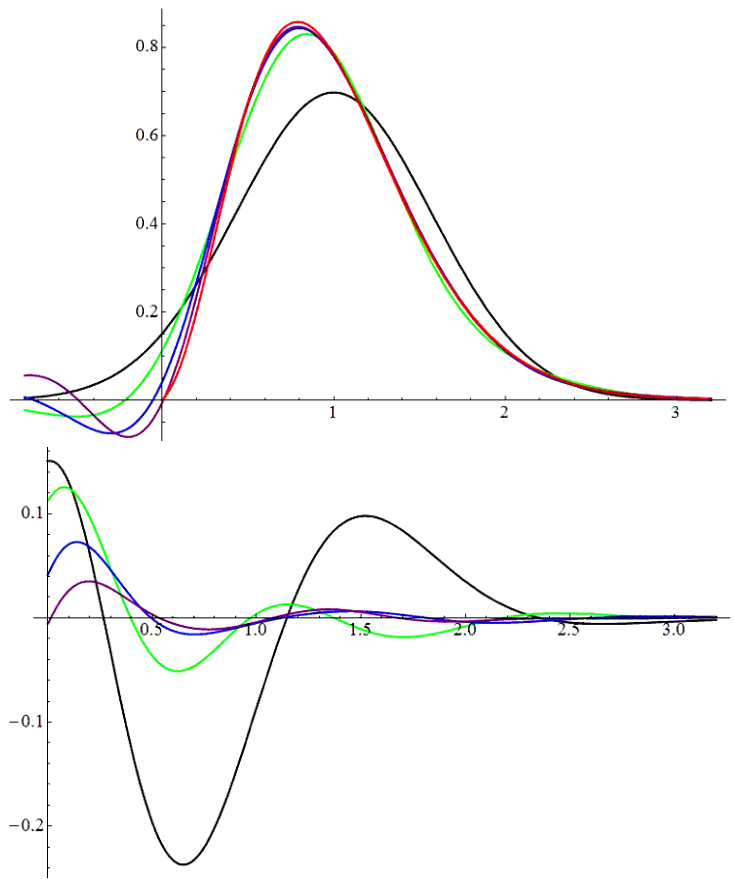

Fig. $2 t=3, \mu=1, \sigma=0.33, \theta=0.1$. (Black, Green, Blue, Purple) lines denote (0th, 1st, 2nd, 3rd) order approximation of asymptotic expansion, respectively. Red line denotes the exact density function given by a non-central chi-squared distribution. The second graph represents the difference from the exact density function. 

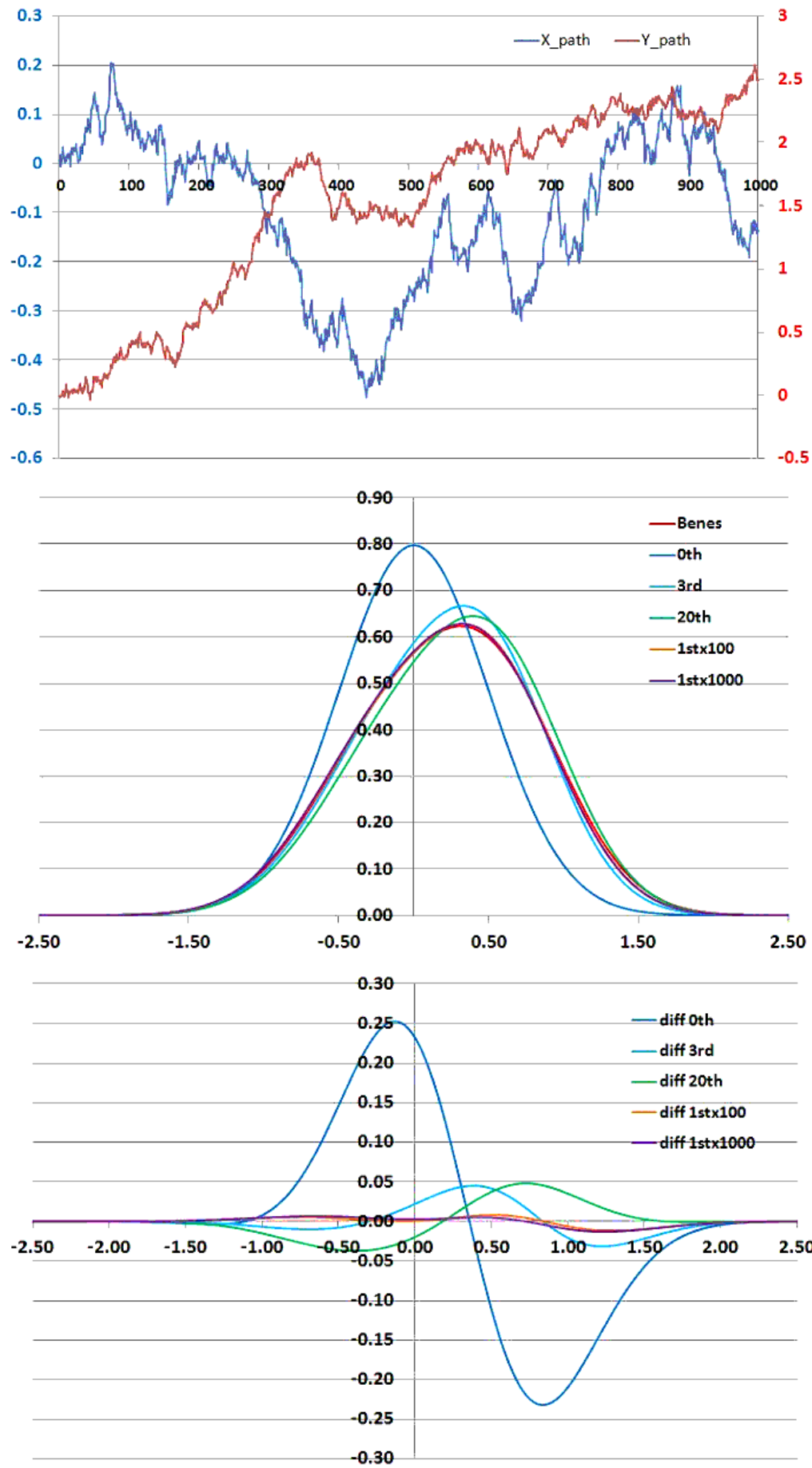

Fig. $3 t=1, d t=10^{-3}, a=0.8, \sigma=0.5, h_{1}=0.8, h_{2}=0.5$ with polynomial function fitted with $w=2.0$. From top to bottom, the sample path, exact and approximated density functions, and the difference of the approximated densities from the exact one. In the middle graph, a red line labeled by "Benes" denotes the exact density function. 

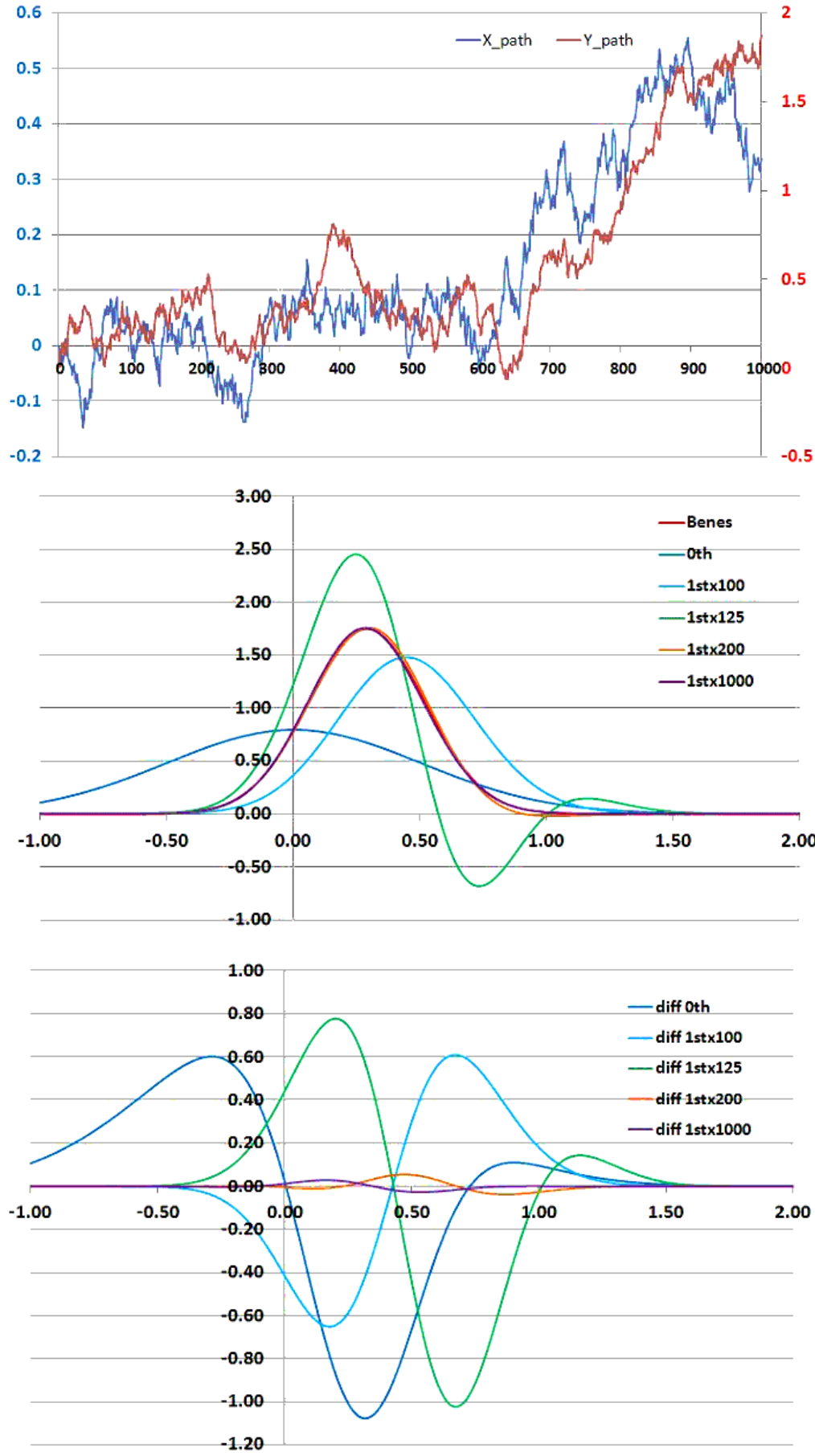

Fig. $4 t=1, d t=10^{-3}, a=0.5, \sigma=0.5, h_{1}=10.0, h_{2}=0.5$ with polynomial function fitted with $w=2.0$. From top to bottom, the sample path, exact and approximated density functions, and the difference of the approximated densities from the exact one. In the middle graph, a red line labeled by "Benes" denotes the exact density function. 

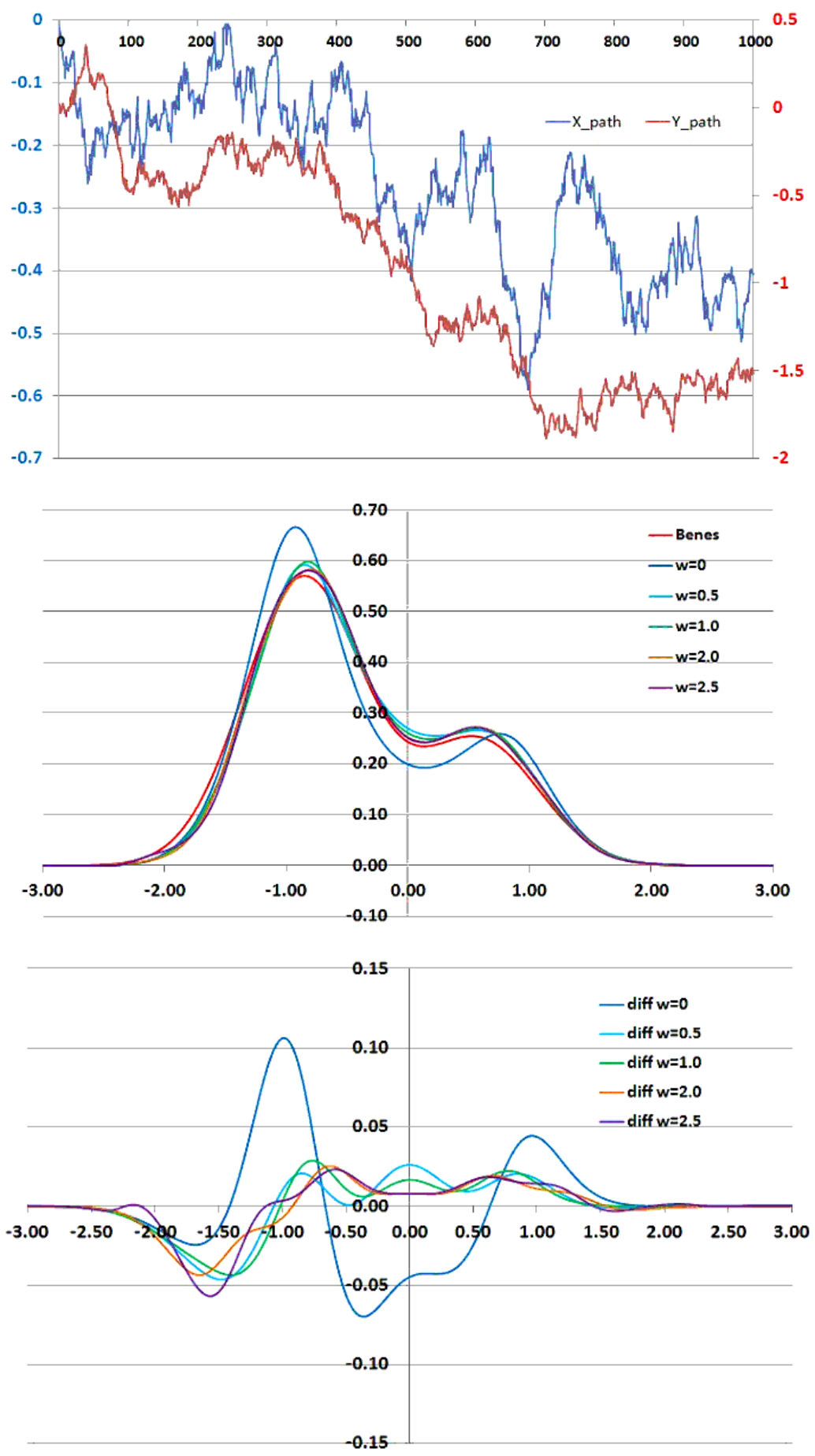

Fig. $5 t=1, d t=10^{-3}, a=1.5, \sigma=0.5, h_{1}=0.7, h_{2}=0.5$ with 1,000 substeps with 1 st order asymptotic expansion. From top to bottom, the sample path, exact and approximated density functions, and the difference of the approximated densities from the exact one. In the middle graph, a red line labeled by "Benes" denotes the exact density function. 


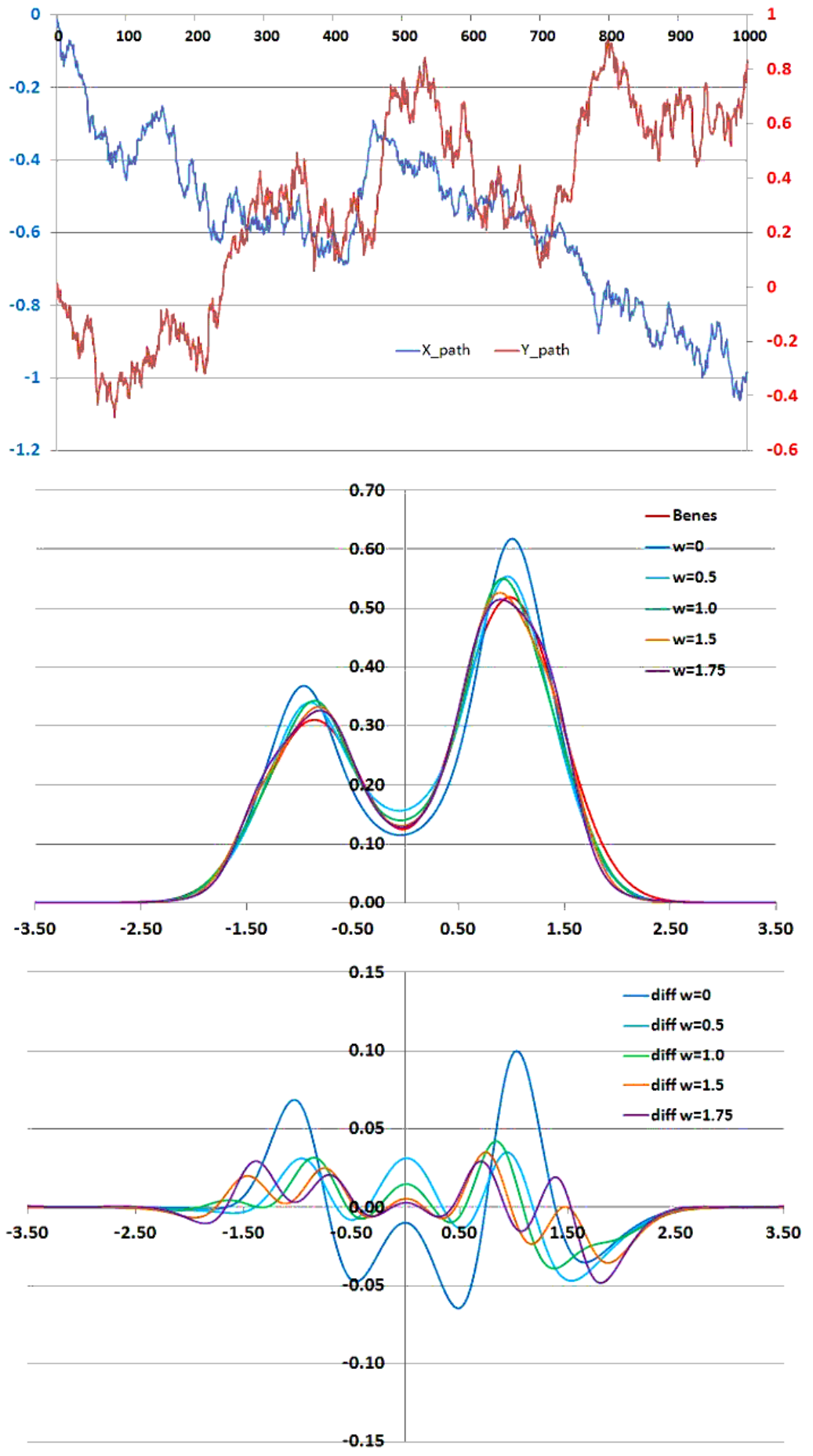

Fig. $6 t=1, d t=10^{-3}, a=2.0, \sigma=0.5, h_{1}=1.0, h_{2}=0.5$ with 1,000 substeps with 1 st order asymptotic expansion. From top to bottom, the sample path, exact and approximated density functions, and the difference of the approximated densities from the exact one. In the middle graph, a red line labeled by "Benes" denotes the exact density function. 